\title{
Fatores associados ao consumo de sal na população adulta brasileira: Pesquisa Nacional de Saúde
}

\author{
Factors associated with salt intake in the Brazilian adult population: \\ National Health Survey
}

José Geraldo Mill (http://orcid.org/0000-0002-0987-368X) ${ }^{1}$

Deborah Carvalho Malta (https://orcid.org/0000-0002-8214-5734) ${ }^{2}$

Eduardo Augusto Fernandes Nilson (https://orcid.org/0000-0002-2650-4878) ${ }^{3}$

Ísis Eloah Machado (https://orcid.org/0000-0002-4678-2074) ${ }^{4}$

Patrícia Constante Jaime (https://orcid.org/0000-0003-2291-8536) ${ }^{5}$

Regina Tomie Ivata Bernal (https://orcid.org/0000-0002-7917-3857) ${ }^{6}$

Laís Santos de Magalhães Cardoso (https://orcid.org/0000-0002-1114-5470) ${ }^{6}$

Célia Landman Szwarcwald (http://orcid.org/0000-0002-7798-2095) ${ }^{7}$

${ }^{1}$ Centro de Ciências da Saúde, Departamento de Ciências Fisiológicas, Universidade Federal do Espírito Santo. Av. Marechal Campos 1.468, Maruípe. 29040-090 Vitória ES Brasil. josegmill@gmail.com ${ }^{2}$ Departamento de Enfermagem Materno Infantil e Saúde Pública, Escola de Enfermagem, Universidade Federal de Minas Gerais. Belo Horizonte MG Brasil. ${ }^{3}$ Secretaria de Vigilância em Saúde, Ministério da Saúde. Brasília DF Brasil.

${ }^{4}$ Programa de Pós-Graduação em Saúde e Nutrição, Departamento de Medicina de Família, Universidade Federal de Ouro Preto. Ouro Preto MG Brasil.

${ }^{5}$ Núcleo de Pesquisas Epidemiológicas em Nutrição e Saúde, Departamento de Nutrição, Faculdade de Saúde Pública, Universidade de São Paulo. São Paulo SP Brasil.

${ }^{6}$ Programa de Pós-Graduação em Enfermagem, Escola de Enfermagem, Universidade Federal de Minas Gerais. Belo Horizonte MG Brasil. ${ }^{7}$ Instituto de Comunicação e Informação Científica e Tecnológica em Saúde, Fundação Oswaldo Cruz. Rio de Janeiro RJ Brasil.
Abstract This paper aims to identify the factors associated with high salt intake in the Brazilian adult population. This is a cross-sectional study with 8,083 adults participating in the National Health Survey (PNS, 2014/15). Salt intake was based on the estimation of 24-hour urinary sodium calculated from the sodium/creatinine ratio in spot urine samples. The highest quartile of the distribution was considered high salt intake. The relationship between high salt consumption and sociodemographic factors, lifestyles, morbidity, and self-rated health status was analyzed by calculating the crude prevalence ratios and the prevalence ratios adjusted for age and gender. Approximately $28.1 \%$ had an estimated salt intake higher than $10.56 \mathrm{~g} /$ day. Overweight (Adjusted Prevalence Ratio; 95\%CI - PRadj 1.23; 1.091.39), obesity (PRadj 1.61; 1.43-1.83), and diabetes (PRadj 1.36; 1.17-1.58) were positively associated with high salt intake. Female gender (PRadj 0.73; 0.66-0.80), high schooling level (PRadj 0.88; 0.79-0.99), living in the North and chronic kidney disease (PRadj 0.71; 0.56-0.90) were protective factors. Salt consumption is elevated nationwide and in all population subgroups, requiring coordinated actions.

Key words Dietary Sodium, Feeding Behavior, Health Surveys, Urine
Resumo $O$ objetivo deste artigo é identificar os fatores associados ao consumo elevado de sal na população brasileira adulta. Estudo transversal com dados de 8.083 adultos da Pesquisa Nacional de Saúde (PNS, 2014/15). O consumo de sal foi baseado na estimativa de excreção urinária de sódio de 24 horas, calculada pela relação sódio/creatinina em amostra de urina casual. Considerou-se consumo elevado o quartil mais alto da distribuição. A relação entre consumo elevado de sal e fatores sociodemográficos, estilos de vida, morbidade e autoavaliação do estado de saúde foi analisada pelo cálculo das razões de prevalência brutas e ajustadas por idade e sexo. 28,1\% apresentavam consumo estimado de sal maior que 10,56 g/dia. Estiveram positivamente associados ao consumo elevado de sal a presença de sobrepeso (Razão de Prevalência ajustada; IC95\% - RPaj 1,23; 1,091,39), obesidade (RPaj 1,61; 1,43-1,83) e diabetes (RPaj 1,36; 1,17-1,58). Foram fatores de proteção o sexo feminino ( $R P a j 0,73 ; 0,66-0,80$ ), escolaridade elevada (RPaj 0,88; 0,79-0,99), morar na região Norte e presença de doença renal crônica (RPaj 0,71; 0,56-0,90). O consumo de sal é elevado em todo o país e em todos os subgrupos da população, demandando ações coordenadas para seu enfrentamento.

Palavras-chave Sódio na Dieta, Comportamento Alimentar, Inquéritos Epidemiológicos, Urina 


\section{Introdução}

Apesar da quase onipresença atual do sal na alimentação, sua introdução na dieta é relativamente recente na evolução humana, provavelmente iniciada com a descoberta de suas propriedades na conservação dos alimentos. Os humanos, desde seus ancestrais, evoluíram por milhões de anos com uma dieta de menos de $0,1 \mathrm{~g} /$ dia de sódio $(0,25 \mathrm{~g} /$ dia de $\mathrm{NaCl})$, quantidade esta naturalmente existente nos alimentos ${ }^{1}$. $\mathrm{O}$ sal de adição somente passou a fazer parte da dieta nos últimos 5.000 a 10.000 anos $^{2}$. Assim, o consumo excessivo de sal, e consequentemente de sódio, representa um grande desafio aos sistemas fisiológicos tendo em vista a necessidade contínua de eliminar pelos rins o sódio ingerido na dieta ${ }^{3}$.

O consumo excessivo de sal é considerado o principal fator de risco dietético para as Doenças Crônicas Não Transmissíveis (DCNT), particularmente por sua relação com os desfechos cardiovasculares mediados pela hipertensão arterial (HA), como acidente vascular cerebral, infarto do miocárdio, miocardiopatia hipertensiva e doença renal crônica ${ }^{2,4}$. Além desses, outros desfechos como câncer de estômago, osteoporose e obesidade também se associam ao consumo excessivo de sódio ${ }^{4}$. Globalmente, estima-se que o consumo excessivo de sódio esteja associado a cerca de 3 milhões de mortes e perda de 70 milhões de DALYs (Disability-Adjusted Life Years - Anos de Vida Ajustados por Incapacidade $)^{5}$.

Em termos globais, estima-se que mais de 95\% das populações consomem sódio em excesso (em média $3,9 \mathrm{~g} /$ dia, variando de 2,2 g a 5,5 g/ dia), enquanto a recomendação da Organização Mundial da Saúde (OMS) é de até 2 g de sódio/ dia, equivalente a $5 \mathrm{~g}$ de sal de cozinha ${ }^{6}$.

As primeiras estimativas de consumo de sal/ sódio da população brasileira foram obtidas em 2002-2003 por metodologias indiretas, baseadas na aquisição domiciliar de alimentos ${ }^{7}$, e em 20082009 por meio de recordatórios alimentares de 24 horas $^{8}$. As estimativas a partir da aquisição domiciliar tendem a superestimar o consumo, enquanto os recordatórios podem subestimá-lo; contudo são importantes para a identificação das fontes dietéticas de sódio 9 . Nesse sentido, a principal fonte dietética de sódio dos brasileiros é o sal de adição, incluindo o sal usado no preparo dos alimentos $(74 \%)$ e o sódio contido nos alimentos industrializados $(19 \%)^{10}$.

Em 2013, foi realizada a primeira avaliação direta do consumo de sal na população na Pesquisa Nacional de Saúde (PNS). Para tanto, foi obtida a relação sódio/creatinina em amostra de urina casual. Estimou-se consumo médio de 9,34 g de sal por dia. Mais de 95\% da população adulta apresentou consumo excessivo de sal $(>5$ g/dia), independentemente de sexo, grupo etário, escolaridade, cor da pele e região geográfica ${ }^{11}$. $\mathrm{O}$ estudo mostrou também grande variabilidade interindividual, com estimativas de consumo diário de 1 até cerca de $25 \mathrm{~g} /$ dia.

Os fatores que afetam o consumo individual de sal são ainda pouco conhecidos na população brasileira. Sabe-se que, apesar do reconhecimento amplo dos efeitos nocivos do consumo excessivo de sal, principalmente em relação aos seus efeitos na pressão arterial, há baixo conhecimento dos níveis adequados de ingestão, tanto no Brasil como em outros países da América Latina ${ }^{12}$. Além disso, no Brasil, a autopercepção do consumo de sal é bastante distorcida, sendo que, na PNS, somente $14,2 \%$ avaliaram seu consumo como elevado ${ }^{13}$.

A redução do consumo de sal é uma das metas do Plano de Ações Estratégicas para o Enfrentamento das DCNT no Brasil (2011-2022) ${ }^{14}$ e, globalmente, a OMS estabeleceu a meta de redução de 30\% no consumo de sal até 2025 em todos os países. Dessa forma, o objetivo deste estudo consiste em identificar os fatores associados ao consumo de sal na população brasileira adulta, o que pode contribuir para o planejamento de ações para alcance dessas metas.

\section{Métodos}

Trata-se de estudo transversal analítico com base nos dados dos exames laboratoriais da PNS coletados em 2014/15. A PNS é uma pesquisa de âmbito nacional e de base domiciliar realizada pelo Instituto Brasileiro de Geografia e Estatística, mediante parceria com o Ministério da Saúde. A pesquisa utilizou amostra probabilística em três estágios. Foram feitas entrevistas com um morador adulto ( $>18$ anos) de 64.348 domicílios residenciais. Detalhes metodológicos adicionais foram anteriormente descritos ${ }^{15,16}$.

Da amostra da PNS foi separada uma subamostra aleatória de 8.952 indivíduos com coleta de sangue e urina, tendo sido excluídas desta análise 869 amostras com material insuficiente, perdas e outros ${ }^{15}$. Assim, a análise atual refere-se a 8.083 participantes. O estudo adotou pesos de pós-estratificação segundo sexo, idade, escolaridade e região, visando estabelecer estimativas para a população adulta brasileira ${ }^{15}$. 
As amostras de urina foram coletadas no domicílio em diferentes horários do dia. Uma alíquota de $5 \mathrm{~mL}$ foi transferida para um frasco estéril acondicionado em bolsa térmica e armazenado em geladeira até envio para o laboratório central em São Paulo. A dosagem do sódio urinário foi feita por eletrodo seletivo e a dosagem de creatinina pelo método de Jaffé sem desproteinização ${ }^{15}$.

A excreção urinária de sódio de 24 horas foi estimada pela equação de Tanaka et al..$^{17}$, que foi validada para a população brasileira em estudo piloto da $\mathrm{PNS}^{18}$. Para uso desta equação, é necessário estimar a excreção urinária (Ur) de creatinina $(\mathrm{Cr})$ prevista para 24 horas $(\mathrm{CrPr} 24 \mathrm{~h}$, em $\mathrm{mg}$ ), a partir da idade (anos), peso (kg) e estatura $(\mathrm{cm})$ do indivíduo, obtidos na visita domiciliar, segundo abaixo:

CrPr 24h, $m g=[(14,89 x$ peso, $k g)+(16,14 x$

estatura, cm) - (2,04x idade, anos) ] - 2.244,5

Dada a CrPr24h, estima-se a relação sódio/ creatinina na urina em 24 horas (Na Ur 24h, $\mathrm{mEq}$ ) pela equação a seguir:

$$
\begin{aligned}
N a \text { Ur24h, } m E q= & \left(\frac{N a \text { UR casual, } m E q / L \times 10}{\text { CrUr casual, } m g / d L}\right) \\
& x \text { CrPr24h, } m g
\end{aligned}
$$

A estimativa da excreção de sódio em $24 \mathrm{~h}$ (mEq) é dada pela equação:

$$
\mathrm{Na} U r 24 h, m E q=2,98 \times N a U r^{0,398}
$$

O consumo de sal foi estimado considerando-se todo o sódio excretado na urina como sendo ingerido como $\mathrm{NaCl}$ :

$$
\begin{gathered}
\text { Consumo estimado de sal, g/dia }= \\
\text { Na 24h, } m E q \times 58,5
\end{gathered}
$$

Para o estudo atual, considerou-se a estimativa de consumo médio de sal na população brasileira de 9,34 g/dia (intervalo de confiança de 95\% - IC95\%: 9,27-9,41 $)^{11}$. Foi realizada a distribuição da excreção urinária em quartis e optou-se, nesta análise, por considerar como "consumo elevado" os indivíduos situados no quartil mais alto, equivalente ao Percentil 75 ou acima ( $\geq$ P75), ou seja, aqueles com consumo $\geq 10,56 \mathrm{~g}$ de sal/dia, o que corresponde à excreção urinária diária de $4,15 \mathrm{~g}$ de sódio. Foram incluídos na análise os seguintes blocos de variáveis de interesse: a) variáveis sociodemográficas sexo (masculino e feminino), faixa etária ( 18 a 29, 30 a 44, 45 a 59 e 60 ou mais anos), escolaridade (sem instrução até ensino fundamental incompleto, ensino fundamental completo a médio incompleto, ensino médio completo e mais), cor da pele (branca, preta, parda e outra) e macrorregião do país (Norte, Nordeste, Sudeste, Sul e Centro-Oeste); b) variáveis sobre estilos de vida consumo de carne vermelha com gordura (sim ou não), consumo de refrigerantes 5 vezes ou mais dias na semana (sim ou não), consumo de bebida alcoólica 1 vez ou mais por mês (sim ou não), uso de tabaco (sim ou não); c) variáveis relativas a morbidades, adiposidade segundo o Índice de Massa Corporal - IMC (baixo peso ou normal, sobrepeso, obesidade ${ }^{19}$ ), diabetes (hemoglobina glicada - HbAlc $\geq 6,5 \%$ ou uso de hipoglicemiante ${ }^{20,21}$, sim ou não), pressão arterial elevada $\left(\geq 140-90 \mathrm{~mm} \mathrm{Hg}^{22}\right.$, sim ou não), colesterol total elevado (colesterol total $\geq 200 \mathrm{mg} / \mathrm{dL}^{23}$, sim ou não), Doença Renal Crônica - DRC (Taxa de Filtração Glomerular - TFG $<60 \mathrm{~mL} / \mathrm{min} / 1,73 \mathrm{~m}^{224}$, sim ou não, sem utilização de fator de correção para raça negra), anemia (hemoglobina - $\mathrm{Hb}<12 \mathrm{~g} / \mathrm{dL}^{25}$, sim ou não); $\mathrm{d}$ ) auto avaliação do estado de saúde (muito bom e bom, regular, ruim e muito ruim). Foram calculadas as prevalências e intervalos de $95 \%$ de confiança (IC95\%) e realizadas análises bivariadas por meio do teste de qui-quadrado. Calculou-se também as razões de prevalência brutas (RP) e ajustadas (RPaj) segundo idade e sexo pelo método de regressão de Poisson com variância robusta e respectivos IC95\%.

As análises foram realizadas com o auxílio do programa estatístico Data Analysis and Statistical Software (Stata), versão 14, usando-se o comando survey para análise de dados de inquéritos com amostra complexa.

A PNS 2013 foi aprovada pela Comissão Nacional de Ética em Pesquisa (CONEP) do Conselho Nacional de Saúde, Ministério da Saúde. Os participantes da pesquisa assinaram termo de consentimento livre e esclarecido (TCLE) antes da coleta de dados.

\section{Resultados}

A Figura 1 mostra que a curva de consumo segue uma distribuição aproximadamente normal (Figura 1A), com consumo mais elevado no sexo masculino (Figura 1B). Conforme mostra a Tabela 1, 28,14\% (IC95\%: 26,79-29,52) dos participantes situavam-se no quartil mais alto de 
$(1 \mathrm{~A})$

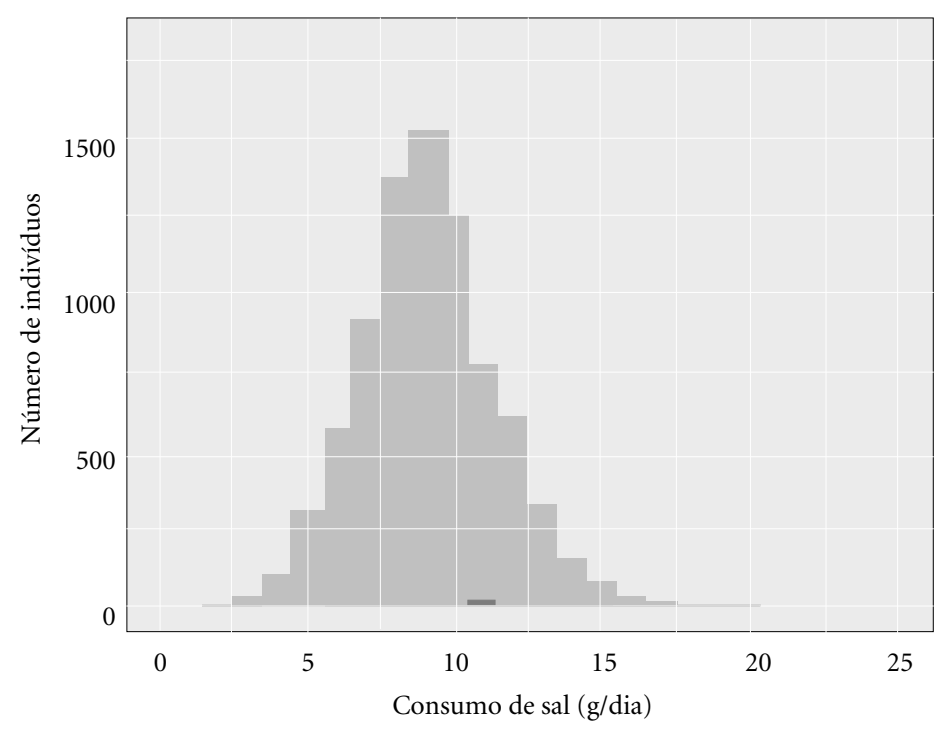

Percentil 75

Maior ou igual

Menor

(1B)

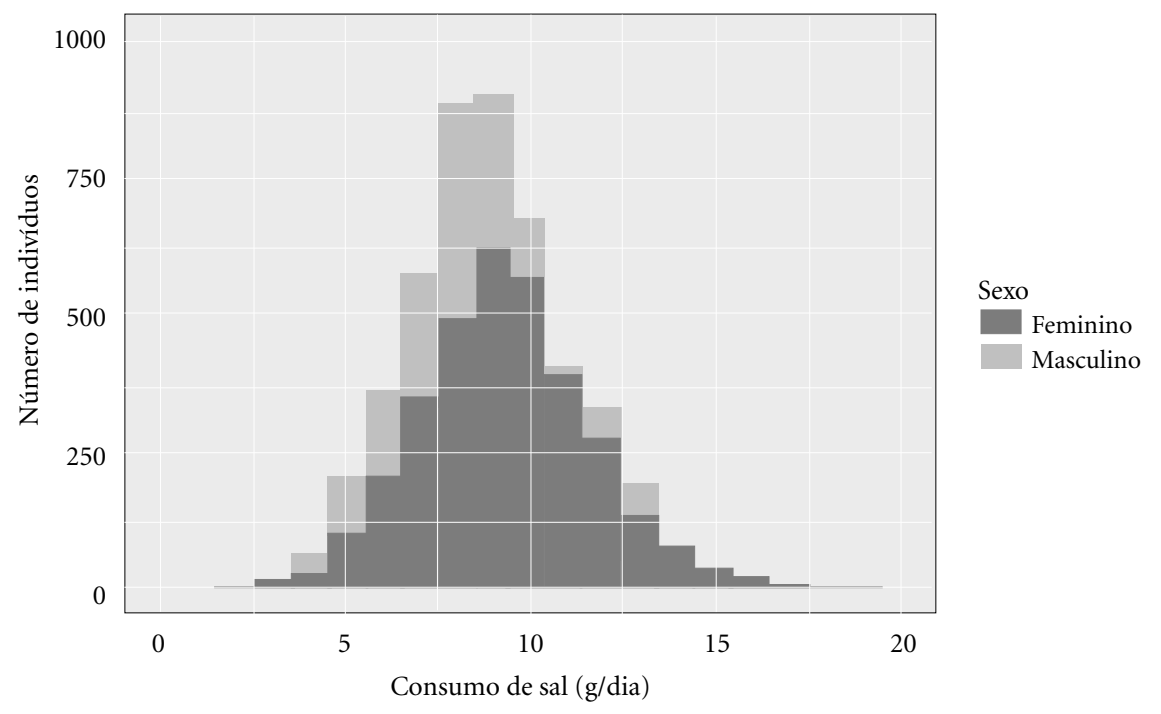

Figura 1. Distribuição da ingestão estimada de sal (g/dia) na população brasileira, PNS 2014-2015 (1A) e distribuição da ingestão estimada de sal (g/dia) em homens e mulheres na população brasileira, PNS 2014-2015 (1B).

Fonte: Elaborado pelos autores.

consumo ( $\geq 10,56 \mathrm{~g}$ de sal diários). Os dados de prevalência com os respectivos IC95\% mostraram que este consumo mais elevado se situou na população masculina (32,84\%; IC95\%: 30,68$35,07)$, nos portadores de sobrepeso $(28,89 \%$; IC95\%: 26,67-31,21) e obesidade (35,84\%; IC95\%: 32,92-38,87), nos que relataram consu- mo de carne vermelha com gordura (30,41\%; IC95\%: 27,84-33,12), portadores de diabetes (34,30\%; IC95\%: 29,88-39,01) e que consumiam bebidas alcoólicas 1 vez ou mais por mês (31,00\%; IC95\%: 28,11-34,04). O consumo elevado de sal foi menor em idosos (24,8\%; IC95\%: 22,21-27,6), na população da região Norte 
Tabela 1. Ingestão estimada de sal (g/dia) na população brasileira, segundo características sociodemográficas, hábitos de vida e comorbidades. PNS, 2014-2015.

\begin{tabular}{|c|c|c|c|c|c|}
\hline \multirow{3}{*}{ Variáveis } & \multicolumn{4}{|c|}{ Ingestão estimada de sal (g/dia) } & \multirow{3}{*}{$\mathbf{p}^{*}$} \\
\hline & \multicolumn{2}{|c|}{$\begin{array}{l}\text { Menor que P75 } \\
(<10.56 \mathrm{~g} / \mathrm{dia})\end{array}$} & \multicolumn{2}{|c|}{$\begin{array}{l}\text { Maior ou igual a P75 } \\
(\geq 10.56 \mathrm{~g} / \mathrm{dia})\end{array}$} & \\
\hline & $\%$ & IC95\% & $\%$ & IC95\% & \\
\hline Total & 71,86 & {$[70,48-73,21]$} & 28,14 & {$[26,79-29,52]$} & \\
\hline \multicolumn{6}{|l|}{ Sexo } \\
\hline Masculino & 67,16 & {$[64,93-69,32]$} & 32,84 & {$[30,68-35,07]$} & 0,00 \\
\hline Feminino & 76,12 & {$[74,43-77,73]$} & 23,88 & {$[22,27-25,57]$} & \\
\hline \multicolumn{6}{|l|}{ Faixa etária } \\
\hline 18 a 29 & 72,16 & {$[68,97-75,15]$} & 27,84 & {$[24,85-31,03]$} & 0,04 \\
\hline 30 a 44 & 69,41 & {$[66,86-71,84]$} & 30,59 & {$[28,16-33,14]$} & \\
\hline 45 a 59 & 72,20 & {$[69,65-74,62]$} & 27,80 & {$[25,38-30,35]$} & \\
\hline 60 anos ou mais & 75,20 & {$[72,40-77,79]$} & 24,80 & {$[22,21-27,60]$} & \\
\hline \multicolumn{6}{|l|}{ Escolaridade } \\
\hline Sem instrução & 70,98 & {$[68,92-72,95]$} & 29,02 & {$[27,05-31,08]$} & 0,13 \\
\hline Fundamental & 69,77 & {$[66,02-73,27]$} & 30,23 & {$[26,73-33,98]$} & \\
\hline Médio Completo ou mais & 73,34 & {$[71,19-75,40]$} & 26,66 & {$[24,60-28,81]$} & \\
\hline \multicolumn{6}{|l|}{ Cor da pele } \\
\hline Branca & 72,18 & {$[70,05-74,22]$} & 27,82 & {$[25,78-29,95]$} & 0,80 \\
\hline Preta & 70,10 & {$[65,10-74,65]$} & 29,90 & {$[25,35-34,90]$} & \\
\hline Parda & 71,82 & {$[69,85-73,71]$} & 28,18 & {$[26,29-30,15]$} & \\
\hline Outra & 74,97 & {$[62,20-84,51]$} & 25,03 & {$[15,49-37,80]$} & \\
\hline \multicolumn{6}{|l|}{ Região } \\
\hline Norte & 79,36 & {$[77,44-81,15]$} & 20,64 & {$[18,85-22,56]$} & 0,00 \\
\hline Nordeste & 74,48 & {$[72,70-76,17]$} & 25,52 & {$[23,83-27,30]$} & \\
\hline Sudeste & 68,41 & {$[65,72-70,99]$} & 31,59 & {$[29,01-34,28]$} & \\
\hline Sul & 73,56 & {$[70,49-76,42]$} & 26,44 & {$[23,58-29,51]$} & \\
\hline Centro-Oeste & 72,66 & {$[69,39-75,72]$} & 27,34 & {$[24,28-30,61]$} & \\
\hline \multicolumn{6}{|c|}{ Consumo de carne vermelha com gordura } \\
\hline Não & 72,80 & {$[71,11-74,42]$} & 27,20 & {$[25,58-28,89]$} & 0,04 \\
\hline Sim & 69,59 & {$[66,88-72,16]$} & 30,41 & {$[27,84-33,12]$} & \\
\hline
\end{tabular}

(20,64\%; IC95\%: 18,85-22,56), nos portadores de doença renal crônica (18,96\%; IC95\%: 15,0423,61). Não ocorreu associação entre a distribuição dos quartis e consumo de sal para diferentes graus de escolaridade, cor da pele, consumo de refrigerantes, tabagismo, pressão arterial elevada aferida na visita domiciliar em que foi feita a coleta de urina, colesterol elevado, anemia e auto avaliação em saúde.

A Tabela 2 apresenta as RP brutas e ajustadas da relação entre o consumo elevado de sal e os fatores estudados. A RPaj por sexo e idade (RPaj; IC95\%) mostrou que o consumo mais elevado de sal ( $\geq$ P75) esteve associado ao sexo masculino, à presença de sobrepeso (RPaj 1,23; IC95\%: 1,09-1,39), obesidade (RPaj 1,61; IC95\%: 1,43-
1,83) e diabetes (RPaj 1,36; IC95\%: 1,17-1,58). Foram identificados como fatores de proteção, isto é, menor consumo de sal, o sexo feminino (RPaj 0,73; IC95\%: 0,66-0,80), escolaridade mais elevada $(0,88$; IC95\%: 0,79-0,99), morar na região Norte, e presença de doença renal crônica (TFG $<60 \mathrm{ml} / \mathrm{min} / 1.73 \mathrm{~m}^{2}$ ) (RPaj 0,71; IC95\%: 0,56-0,90).

\section{Discussão}

O estudo analisou dados da PNS e identificou os fatores demográficos, comportamentais e clínicos associados ao consumo elevado do sal, ( $\geq 10,56 \mathrm{~g} /$ dia), correspondente ao P75 da distribuição des- 
Tabela 1. Ingestão estimada de sal (g/dia) na população brasileira, segundo características sociodemográficas, hábitos de vida e comorbidades. PNS, 2014-2015.

\begin{tabular}{|c|c|c|c|c|c|}
\hline \multirow{3}{*}{ Variáveis } & \multicolumn{4}{|c|}{ Ingestão estimada de sal (g/dia) } & \multirow{3}{*}{$\mathbf{p}^{*}$} \\
\hline & \multicolumn{2}{|c|}{$\begin{array}{c}\text { Menor que P75 } \\
(<10.56 \text { g/dia })\end{array}$} & \multicolumn{2}{|c|}{$\begin{array}{l}\text { Maior ou igual a P75 } \\
\quad(\geq 10.56 \mathrm{~g} / \mathrm{dia})\end{array}$} & \\
\hline & $\%$ & IC95\% & $\%$ & IC95\% & \\
\hline \multicolumn{6}{|c|}{ Consumo de refrigerante 5 ou mais dias na semana } \\
\hline Não & 72,09 & {$[70,55-73,58]$} & 27,91 & {$[26,42-29,45]$} & 0,52 \\
\hline Sim & 70,96 & {$[67,78-73,95]$} & 29,04 & {$[26,05-32,22]$} & \\
\hline \multicolumn{6}{|c|}{ Consumo de bebida alcoólica 1 vez ou mais por mês } \\
\hline Não & 72,80 & {$[71,25-74,30]$} & 27,20 & {$[25,70-28,75]$} & 0,02 \\
\hline Sim & 69,00 & {$[65,96-71,89]$} & 31,00 & {$[28,11-34,04]$} & \\
\hline \multicolumn{6}{|l|}{ Tabagismo } \\
\hline Não & 71,56 & {$[70,07-73,01]$} & 28,44 & {$[26,99-29,93]$} & 0,37 \\
\hline Sim & 73,39 & {$[69,58-76,88]$} & 26,61 & {$[23,12-30,42]$} & \\
\hline \multicolumn{6}{|l|}{ Índice de Massa Corporal } \\
\hline Baixo peso/Normal & 76,58 & {$[74,45-78,59]$} & 23,42 & {$[21,41-25,55]$} & 0,00 \\
\hline Sobrepeso & 71,11 & {$[68,79-73,33]$} & 28,89 & {$[26,67-31,21]$} & \\
\hline Obesidade & 64,16 & {$[61,13-67,08]$} & 35,84 & {$[32,92-38,87]$} & \\
\hline \multicolumn{6}{|c|}{ Diabetes ( $\mathrm{HbA} 1 \mathrm{C} \geq 6,5$ ou uso de medicamento) } \\
\hline Não & 72,31 & {$[70,83-73,74]$} & 27,69 & {$[26,26-29,17]$} & 0,00 \\
\hline Sim & 65,70 & {$[60,99-70,12]$} & 34,30 & {$[29,88-39,01]$} & \\
\hline \multicolumn{6}{|l|}{ Hipertensão arterial aferida } \\
\hline Não & 72,21 & {$[70,74-73,62]$} & 27,79 & {$[26,38-29,26]$} & 0,13 \\
\hline Sim & 68,79 & {$[64,32-72,93]$} & 31,21 & {$[27,07-35,68]$} & \\
\hline \multicolumn{6}{|c|}{ Colesterol total elevado ( $\geq 200 \mathrm{mg} / \mathrm{dL})$} \\
\hline Não & 71,34 & {$[69,57-73,06]$} & 28,66 & {$[26,94-30,43]$} & 0,46 \\
\hline Sim & 72,43 & {$[70,11-74,64]$} & 27,57 & {$[25,36-29,89]$} & \\
\hline \multicolumn{6}{|l|}{ DRC $\left(\right.$ TFG $\left.<60 \mathrm{~mL} / \mathrm{min} / 1.73 \mathrm{~m}^{2}\right)$} \\
\hline Não & 71,07 & {$[69,60-72,50]$} & 28,93 & {$[27,50-30,40]$} & 0,00 \\
\hline $\operatorname{Sim}$ & 81,04 & {$[76,39-84,96]$} & 18,96 & {$[15,04-23,61]$} & \\
\hline \multicolumn{6}{|l|}{ Anemia $(\mathrm{Hb}<12 \mathrm{~g} / \mathrm{dL})$} \\
\hline Não & 71,52 & {$[69,97-73,03]$} & 28,48 & {$[26,97-30,03]$} & 0,54 \\
\hline Sim & 72,81 & {$[68,84-76,44]$} & 27,19 & {$[23,56-31,16]$} & \\
\hline \multicolumn{6}{|l|}{ Auto avaliação do estado de saúde } \\
\hline Muito Bom/Bom & 72,00 & {$[70,22-73,72]$} & 28,00 & {$[26,28-29,78]$} & 0,80 \\
\hline Regular & 71,79 & {$[69,35-74,10]$} & 28,21 & {$[25,90-30,65]$} & \\
\hline Ruim/Muito ruim & 70,17 & {$[65,00-74,88]$} & 29,83 & {$[25,12-35,00]$} & \\
\hline
\end{tabular}

*Valor de p do teste de qui-quadrado.

Fonte: Elaborado pelos autores.

te parâmetro na população brasileira adulta. Em geral, o consumo mais alto esteve associado ao sexo masculino ou à presença de sobrepeso, obesidade ou diabetes, enquanto o consumo mais baixo mostrou associação com a escolaridade mais elevada, morar na região Norte e presença de doença renal crônica.

A PNS possibilitou pela primeira vez estimar o consumo de sal na população brasileira por meio de análise de urina ${ }^{11}$. Os dados apontam consumo elevado generalizado na população, uma vez que apenas 3,4\% desta apresentou consumo recomendado pela OMS ( $<5$ g por dia), o que converge com resultados de outros estudos no Brasil e em outros países ${ }^{11,26-29}$.

O padrão-ouro para estimar o consumo de sal é a coleta urinária de 24 horas. Este método, entretanto, é de difícil realização em estudos populacionais pela grande possibilidade de erros na coleta, o que reduz de forma importante a preci- 
Tabela 2. Razão de Prevalência bruta e ajustada de consumo elevado de sal (quartil mais alto), segundo características sociodemográficas, hábitos de vida e comorbidades na população brasileira. PNS, 2014-2015.

\begin{tabular}{|c|c|c|c|c|c|c|c|c|}
\hline \multirow{3}{*}{ Variáveis } & \multicolumn{8}{|c|}{ Ingestão estimada de sal (g/dia) } \\
\hline & \multicolumn{2}{|c|}{$\begin{array}{l}\text { Maior ou igual a P75 } \\
\quad(\geq 10.56 \mathrm{~g} / \mathrm{dia})\end{array}$} & \multirow{2}{*}{$\mathbf{R P}_{\text {bruta }}$} & \multicolumn{2}{|c|}{$\mathrm{IC}(95 \%)$} & \multirow{2}{*}{$\mathbf{R} \mathbf{P}_{\text {ajustada }}{ }^{*}$} & \multicolumn{2}{|c|}{$\mathrm{IC}(95 \%)$} \\
\hline & $\%$ & IC95\% & & & & & & \\
\hline Total & 28,14 & {$[26,79-29,52]$} & & & & & & \\
\hline \multicolumn{9}{|l|}{$\operatorname{Sexo}^{* *}$} \\
\hline Masculino & 32,84 & {$[30,68-35,07]$} & 1,00 & & & 1,00 & & \\
\hline Feminino & 23,88 & {$[22,27-25,57]$} & 0,73 & 0,66 & 0,80 & 0,73 & 0,66 & 0,80 \\
\hline \multicolumn{9}{|l|}{ Faixa etária ${ }^{\star * *}$} \\
\hline 18 a 29 & 27,84 & {$[24,85-31,03]$} & 1,00 & & & 1,00 & & \\
\hline 30 a 44 & 30,59 & {$[28,16-33,14]$} & 1,10 & 0,96 & 1,26 & 1,11 & 0,97 & 1,27 \\
\hline 45 a 59 & 27,80 & {$[25,38-30,35]$} & 1,00 & 0,87 & 1,15 & 1,01 & 0,87 & 1,16 \\
\hline 60 anos ou mais & 24,80 & {$[22,21-27,60]$} & 0,89 & 0,76 & 1,04 & 0,91 & 0,78 & 1,06 \\
\hline \multicolumn{9}{|l|}{ Escolaridade } \\
\hline Sem instrução & 29,02 & {$[27,05-31,08]$} & 1,00 & & & 1,00 & & \\
\hline Fundamental & 30,23 & {$[26,73-33,98]$} & 1,04 & 0,91 & 1,20 & 1,00 & 0,86 & 1,15 \\
\hline Médio Completo ou mais & 26,66 & {$[24,60-28,81]$} & 0,92 & 0,83 & 1,02 & 0,88 & 0,79 & 0,99 \\
\hline \multicolumn{9}{|l|}{ Cor da pele } \\
\hline Branca & 27,82 & {$[25,78-29,95]$} & 1,00 & & & 1,00 & & \\
\hline Preta & 29,90 & {$[25,35-34,90]$} & 1,08 & 0,90 & 1,28 & 1,07 & 0,90 & 1,27 \\
\hline Parda & 28,18 & {$[26,29-30,15]$} & 1,01 & 0,92 & 1,12 & 1,00 & 0,90 & 1,11 \\
\hline Outra & 25,03 & {$[15,49-37,80]$} & 0,90 & 0,57 & 1,42 & 0,92 & 0,58 & 1,45 \\
\hline \multicolumn{9}{|l|}{ Região } \\
\hline Norte & 20,64 & {$[18,85-22,56]$} & 1,00 & & & 1,00 & & \\
\hline Nordeste & 25,52 & {$[23,83-27,30]$} & 1,24 & 1,10 & 1,38 & 1,25 & 1,11 & 1,40 \\
\hline Sudeste & 31,59 & {$[29,01-34,28]$} & 1,53 & 1,35 & 1,73 & 1,55 & 1,37 & 1,76 \\
\hline Sul & 26,44 & {$[23,58-29,51]$} & 1,28 & 1,11 & 1,48 & 1,30 & 1,12 & 1,50 \\
\hline Centro-Oeste & 27,34 & {$[24,28-30,61]$} & 1,32 & 1,14 & 1,53 & 1,33 & 1,15 & 1,54 \\
\hline
\end{tabular}

são da medida e, consequentemente, a confiabilidade do método. Desta forma, há uma tendência na literatura de se adotar coleta casual para obtenção da relação sódio/creatinina e, por meio de fórmulas, estimar o consumo médio populacional $^{30,31}$. Essa estratégia foi adotada na PNS assim como em outros países ${ }^{32}$, possibilitando maior adesão e rapidez na obtenção dos dados. A opção pela aplicação da equação de Tanaka decorreu do fato de que esta mostrou melhor desempenho em estudo de validação ${ }^{18}$, o que também se verificou em outras populações ${ }^{32}$. Em estudo de validação ${ }^{18}$ foi demonstrado que a fórmula de Tanaka estima o consumo de sal com acurácia de $1 \mathrm{~g} / \mathrm{dia}$, apresentando bom desempenho para se estimar o consumo médio de grandes grupos. Entretanto, a acurácia é baixa nos extremos. Por isso, na prática clínica, a recomendação ainda permanece de se medir o consumo de sal pela coleta urinária de 24 horas ou, alternativamente, com coletas de $12 \mathrm{~h}$ no período da noite, quando tendem a ser menores os erros de coleta ${ }^{31,33}$.

Estudos apontam a associação entre o alto consumo de sal e hipertensão arterial ${ }^{34}$ e doenças cardiovasculares ${ }^{35}$. Entretanto, no estudo atual, a hipertensão arterial não se mostrou associada ao consumo elevado de sal. Isso pode ter ocorrido em função do desenho do estudo, de caráter transversal, ou do fato de que a pressão arterial não foi considerada como variável contínua na análise e sim em relação à proporção de indivíduos em quartis. Além disso, o consumo elevado de forma geral na população brasileira pode dificultar a detecção de uma associação que efetivamente existe. Entretanto, as evidências da associação do consumo de sódio e hipertensão estão 
Tabela 2. Razão de Prevalência bruta e ajustada de consumo elevado de sal (quartil mais alto), segundo características sociodemográficas, hábitos de vida e comorbidades na população brasileira. PNS, 2014-2015.

\begin{tabular}{|c|c|c|c|c|c|c|c|c|}
\hline \multirow{3}{*}{ Variáveis } & \multicolumn{8}{|c|}{ Ingestão estimada de sal (g/dia) } \\
\hline & \multicolumn{2}{|c|}{$\begin{array}{l}\text { Maior ou igual a P75 } \\
\quad(\geq 10.56 \mathrm{~g} / \mathrm{dia})\end{array}$} & \multirow{2}{*}{$\mathbf{R} \mathbf{P}_{\text {bruta }}$} & \multirow{2}{*}{\multicolumn{2}{|c|}{$\mathrm{IC}(95 \%)$}} & \multirow{2}{*}{$\mathbf{R} \mathbf{P}_{\text {ajustada }}$ * } & \multicolumn{2}{|c|}{ IC $(95 \%)$} \\
\hline & $\%$ & IC95\% & & & & & & \\
\hline \multicolumn{9}{|c|}{$\begin{array}{l}\text { Consumo de carne vermelha com } \\
\text { gordura }\end{array}$} \\
\hline Não & 27,20 & {$[25,58-28,89]$} & 1,00 & & & 1,00 & & \\
\hline Sim & 30,41 & {$[27,84-33,12]$} & 1,12 & 1,01 & 1,24 & 1,04 & 0,93 & 1,16 \\
\hline \multicolumn{9}{|c|}{$\begin{array}{l}\text { Consumo de refrigerante } 5 \text { ou mais } \\
\text { dias na semana }\end{array}$} \\
\hline Não & 27,91 & {$[26,42-29,45]$} & & & & 1,00 & & \\
\hline Sim & 29,04 & {$[26,05-32,22]$} & 1,04 & 0,92 & 1,17 & 1,02 & 0,91 & 1,15 \\
\hline \multicolumn{9}{|c|}{$\begin{array}{l}\text { Consumo de bebida alcoólica } 1 \text { vez } \\
\text { ou mais por mês }\end{array}$} \\
\hline Não & 27,20 & {$[25,70-28,75]$} & 1,00 & & & 1,00 & & \\
\hline Sim & 31,00 & {$[28,11-34,04]$} & 1,14 & 1,02 & 1,27 & 1,03 & 0,91 & 1,15 \\
\hline \multicolumn{9}{|l|}{ Tabagismo } \\
\hline Não & 28,44 & {$[26,99-29,93]$} & 1,00 & & & 1,00 & & \\
\hline $\operatorname{Sim}$ & 26,61 & {$[23,12-30,42]$} & 0,94 & 0,81 & 1,08 & 1,12 & 0,97 & 1,29 \\
\hline \multicolumn{9}{|l|}{ Índice de Massa Corporal } \\
\hline Baixo peso/Normal & 23,42 & {$[21,41-25,55]$} & 1,00 & & & 1,00 & & \\
\hline Sobrepeso & 28,89 & {$[26,67-31,21]$} & 1,23 & 1,10 & 1,39 & 1,23 & 1,09 & 1,39 \\
\hline Obesidade & 35,84 & {$[32,92-38,87]$} & 1,53 & 1,36 & 1,73 & 1,61 & 1,43 & 1,83 \\
\hline \multicolumn{9}{|c|}{$\begin{array}{l}\text { Diabetes (HbA1C } \geq 6,5 \text { ou uso de } \\
\text { medicamento) }\end{array}$} \\
\hline Não & 27,69 & {$[26,26-29,17]$} & 1,00 & & & 1,00 & & \\
\hline Sim & 34,30 & {$[29,88-39,01]$} & 1,24 & 1,07 & 1,43 & 1,36 & 1,17 & 1,58 \\
\hline \multicolumn{9}{|c|}{ Hipertensão arterial aferida } \\
\hline Não & 27,79 & {$[26,38-29,26]$} & 1,00 & & & 1,00 & & \\
\hline Sim & 31,21 & {$[27,07-35,68]$} & 1,12 & 0,97 & 1,30 & 1,11 & 0,95 & 1,29 \\
\hline \multicolumn{9}{|c|}{ Colesterol total elevado ( $\geq 200 \mathrm{mg} / \mathrm{dL})$} \\
\hline Não & 28,66 & {$[26,94-30,43]$} & 1,00 & & & 1,00 & & \\
\hline $\operatorname{Sim}$ & 27,57 & {$[25,36-29,89]$} & 0,96 & 0,87 & 1,07 & 0,99 & 0,89 & 1,09 \\
\hline \multicolumn{9}{|c|}{$\mathrm{DRC}\left(\mathrm{TFG}<60 \mathrm{~mL} / \mathrm{min} / 1.73 \mathrm{~m}^{2}\right)$} \\
\hline Não & 28,93 & {$[27,50-30,40]$} & 1,00 & & & 1,00 & & \\
\hline $\operatorname{Sim}$ & 18,96 & {$[15,04-23,61]$} & 0,66 & 0,52 & 0,83 & 0,71 & 0,56 & 0,90 \\
\hline \multicolumn{9}{|l|}{ Anemia $(\mathrm{Hb}<12 \mathrm{~g} / \mathrm{dL})$} \\
\hline Não & 28,48 & {$[26,97-30,03]$} & 1,00 & & & 1,00 & & \\
\hline $\operatorname{Sim}$ & 27,19 & {$[23,56-31,16]$} & 0,95 & 0,82 & 1,11 & 0,97 & 0,84 & 1,13 \\
\hline \multicolumn{9}{|c|}{ Auto avaliação do estado de saúde } \\
\hline Muito Bom/Bom & 28,00 & {$[26,28-29,78]$} & 1,00 & & & 1,00 & & \\
\hline Regular & 28,21 & {$[25,90-30,65]$} & 1,01 & 0,91 & 1,12 & 1,06 & 0,95 & 1,18 \\
\hline Ruim/Muito ruim & 29,83 & {$[25,12-35,00]$} & 1,07 & 0,89 & 1,27 & 1,17 & 0,98 & 1,40 \\
\hline
\end{tabular}

bem estabelecidas na literatura e permanecem as orientações quanto à aplicação de ações de prevenção, visando redução de consumo e de eventos ligados às DCNT ${ }^{36-38}$.
Este estudo apontou associação a um menor consumo de sal nos indivíduos com DRC (TFG $\leq 60 \mathrm{ml} / \mathrm{min} / 1.73 \mathrm{~m}^{2}$ ), calculada pela fórmula de CKD-EPI (Chronic Kidney Disease Epidemiology 
Collaboration $)^{24}$. Ressalta-se que nesta condição já está estabelecido que a redução do consumo de sal é necessária para se evitar o aparecimento de edemas e diminuir a sobrecarga renal ${ }^{39}$. Assim, esse dado sugere adesão, pelo menos em parte dos indivíduos, à recomendação da redução da ingestão de sal nestas condições. Ao contrário, não se detectou diferença no consumo de sal nos indivíduos que apresentaram valores pressóricos elevados no domicílio. Uma das recomendações essenciais em hipertensos é a redução do sal na alimentação, tendo em vista tanto reduzir a pressão como aumentar a resposta farmacológica aos antihipertensivos. Esse achado pode ser explicado pelo fato de que, em estudos transversais, hipertensos apresentam maior excreção urinária de sódio em relação aos normotensos ${ }^{40}$. Esse dado indica baixa adesão a esta recomendação dietética entre os portadores de pressão arterial alta.

O elevado consumo de sal detectado neste estudo já havia sido observado anteriormente na POF 2002-20037. Análises da POF apontaram que a principal fonte de sódio provém do sal adicionado no preparo dos alimentos ${ }^{7,8}$. No Brasil, ocorreu redução progressiva na aquisição domiciliar anual de sal (refinado ou grosso), de 2,98 para 2,47 kg per capita ${ }^{7}$. Entretanto, este fato pode não ter afetado o consumo individual, em decorrência do aumento do consumo de alimentos processados e ultraprocessados ${ }^{41}$, que contêm teores elevados de sal, além do aumento da participação da alimentação fora do domicílio no comportamento dietético da população ${ }^{41,42}$. Desta forma, pode-se considerar não ter havido redução substancial do consumo de sal na população brasileira desde a POF de 2002-2003. Contudo, a variação do consumo só poderá ser determinada em estudos seriados e com uso de mesma metodologia de aferição do consumo deste nutriente ${ }^{31}$.

Pesquisas autorreferidas, como a Vigilância dos Fatores de Risco e Proteção para Doenças Crônicas por Inquérito Telefônico (VIGITEL), mostraram que homens apresentam indicadores dietéticos menos saudáveis do que mulheres, inclusive menor percepção do consumo elevado de sal $^{13}$. Também, consumo mais elevado de sal entre os mais jovens já foi descrito em análises anteriores ${ }^{11}$. Ao se ajustar por escolaridade, entretanto, essas diferenças desapareceram, mostrando que, na população brasileira em geral, o consumo muito elevado de sal está distribuído em todas as faixas etárias.

A literatura aponta piores indicadores de qualidade de dieta em indivíduos com escolaridade mais baixa ${ }^{11,43}$, sendo a escolaridade considerada proxy de condição socioeconômica. Maior escolaridade resulta em maior acesso à informação e exames preventivos e a diagnósticos médicos mais precoces, o que resultaria em melhores indicadores de saúde, corroborando outros dados encontrados na $\mathrm{PNS}^{38}$.

O menor consumo de sal na região Norte deve ser analisado e confrontado com os dados da POF, mas pode ser explicado pelo menor consumo de alimentos ultraprocessados nesta região (11,4\% das calorias, enquanto a média nacional é de $18,4 \%$ ), com elevada proporção de população rural e ribeirinha ainda mantendo estilo de alimentação mais tradicional ${ }^{44}$.

Este estudo revelou associação direta entre acúmulo de gordura corporal e maior consumo de sal. Nesse sentido, estudos mostram a associação entre consumo elevado de sódio e obesidade mediante o aumento do consumo de bebidas adoçadas, que também contêm sódio em sua composição ${ }^{45}$. Mas, também, pode haver relação entre consumo de sódio e obesidade independente da energia consumida, por mecanismos fisiológicos e metabólicos ainda pouco conheci$\operatorname{dos}^{2,46}$.

Outro achado do estudo foi a associação entre diabetes e consumo elevado de sal. Estudos mostram que a redução na ingestão de sódio pode atenuar a resistência à insulina (RI) e induzir alterações em lipoproteínas séricas e marcadores de inflamação semelhantes aos encontrados na síndrome metabólica $(\mathrm{SM})^{47}$. Meta-análise aponta efeitos benéficos da restrição moderada de sódio da dieta no controle do diabetes ${ }^{48}$. Portanto, esperava-se o oposto do encontrado no atual estudo, o que demanda novas investigações. Há que se questionar o apelo da indústria para que pacientes com diabetes prefiram alimentos com rótulos de diet ou light, que possuem maior quantidade de sódio na sua composição, e pautar a reformulação dos alimentos ultraprocessados ${ }^{49,50}$. Assim, refrigerantes, adoçantes, e outros, que são muito consumidos por diabéticos, poderiam contribuir para o consumo elevado de sódio por estes pacientes e explicar os dados aqui encontrados. Evidências recentes apontam que em alguns países pode haver maior consumo de sódio em indivíduos diabéticos, particularmente quando são também hipertensos ${ }^{51}$. Ainda, estudos apontam que há crescente consumo de adoçantes não-calóricos por populações em todo o mundo e que diabéticos são os maiores consumidores de adoçantes artificiais ${ }^{52}$. Apontam, também, que o consumo elevado de bebidas adoçadas artificial- 
mente, que também contribuem para a ingestão do sódio por meio dos edulcorantes à base deste mineral, está associado ao maior risco de acidente vascular cerebral ${ }^{53}$.

Este estudo aponta consumo elevado de sal de forma generalizada na população brasileira e, portanto, indica que os programas de redução de consumo devem ser dirigidos a toda a população. Dentre as iniciativas, destacam-se o Plano Nacional de Enfrentamento das DCNT 2011 14,54, o Plano de Ação Global de DCNTs da OMS ${ }^{55}$, que contém metas de redução do teor de sódio, e o Guia Alimentar para a População Brasileira, que apresenta informações e orientações sobre escolha, preparo e consumo de alimentos (incluindo o uso de pequenas quantidades de ingredientes culinários, como sal, açúcar, óleos e gorduras) e reforça a narrativa de valorização do consumo de alimentos frescos e de procedência regional, alertando para os riscos associados ao consumo de alimentos ultraprocessados, decorrentes dos elevados teores de sódio, gorduras e açúcares ${ }^{56}$. Nessa perspectiva, a realização de acordos voluntários (termos de compromisso) com segmentos do setor produtivo envolvidos na cadeia industrial de alimentos mostrou uma redução do teor de sódio na maioria dos alimentos industrializados avaliados ${ }^{41}$. Entretanto, os dados aqui analisados apontam que estamos longe de atingir as metas voluntárias de redução do consumo de sódio. Parece necessário, portanto, avançar nas medidas de regulação e controle de alimentos, a exemplo da rotulagem nutricional frontal com advertência, regulação da venda e publicidade de alimentos ultraprocessados e taxação de alimentos e bebidas não saudáveis ${ }^{43}$.

Dentre as limitações do presente estudo, destaca-se que não foi utilizado o método padrão -ouro para a estimar o consumo de sódio. Entretanto, foi utilizado um método de menor custo e de mais fácil utilização em inquéritos populacionais, já validado para a população brasileira em estudo anterior ${ }^{18}$. Destaca-se, ainda, que devido ao excessivo consumo de sal pela população brasileira, a categoria de referência para o consumo utilizada também contém indivíduos que consomem além dos $5 \mathrm{~g}$ de sódio por dia recomendados pela $\mathrm{OMS}^{6}$. Este fato pode ter influenciado os resultados aqui apresentados, reduzindo a força de associação entre o consumo elevado de sódio e os fatores estudados. Entretanto, ressalta-se que, ainda sim, foram observados resultados estatisticamente significativos, o que reforça o valor dos achados.

O presente estudo aponta que o consumo de sal é elevado em todo o país e em todos os subgrupos da população, demandando ações coordenadas para seu enfrentamento, como a melhoria do acesso a alimentos saudáveis, a implementação de ações de educação para a saúde, além da regulação e do monitoramento de acordos celebrados com a indústria de alimentos. 


\section{Colaboradores}

DC Malta, JG Mill, RTI Bernal participaram da concepção, delineamento, análise e interpretação dos dados. DC Malta redigiu a primeira versão do artigo. RTI Bernal realizou a análise estatística. EAF Nilson, IE Machado, PC Jaime, LSM Cardoso, CL Szwarcwald realizaram a análise de dados e a revisão crítica do manuscrito. Todos os autores aprovaram a versão a ser publicada.

\section{Financiamento}

Ministério da Saúde, TED 147/2018 (Laboratório da Pesquisa Nacional de Saúde).

\section{Referências}

1. Eaton SB, Konner M. Paleolithic Nutrition - A consideration of its Nature and current implications. $N$ Engl J Med 1985; 312(5):283-289.

2. He FJ, MacGregor GA. Dietary salt, high blood pressure and other harmful effects on health. In: Kilcast D, Angus F, editores. Reducing salt in foods: Practical strategies. Boca Raton: Woodhead Publishing Limited, CRC Press; 2007. p. 18-54.

3. Henney JE, Taylor CL, Boon CS, editores. Strategies to reduce sodium intake in the United States/Committee on Strategies to Reduce Sodium Intake, Food and Nutrition Board. Washington: National Academy of Sciences; 2010.

4. He FJ, Tan M, Ma Y, MacGregor GA. Salt reduction to prevent hypertension and cardiovascular disease: JACC State-of-the-Art Review. J Am Coll Cardiol 2020; 75(6):632-647.

5. GBD 2017 Diet Collaborators. Health effects of dietary risks in 195 countries, 1990-2017: a systematic analysis for the Global Burden of Disease Study 2017. Lancet 2019; 393(19):1958-1972.

6. Powles J, Fahimi S, Micha R, Khatibzadeh S, Shi P, Ezzati M, Engell RE, Lim SS, Danaei G, Mozaffarian D, Global Burden of Diseases Nutrition and Chronic Diseases Expert Group (NutriCoDE). Global, regional and national sodium intakes in 1990 and 2010: A systematic analysis of $24 \mathrm{~h}$ urinary sodium excretion and dietary surveys worldwide. BMJ Open 2013; 3(12):e003733.

7. Sarno F, Claro RM, Levy RB, Bandoni DH, Ferreira SRG, Monteiro CA. Estimativa de consumo de sódio pela população brasileira, 2002-2003. Rev Saúde Pública 2009; 43(2):219-225.

8. Araújo MC, Bezerra IN, Barbosa FS, Junger WL, Yokoo EM, Pereira RA, Sichieri R. Consumo de macronutrientes e ingestão inadequada de micronutrientes em adultos. Rev Saúde Pública 2013; 47(Supl. 1):177s-189s

9. Pan American Health Organization (PAHO/WHO). Salt-Smart Americas: A Guide for Country-Level Action. Washington: PAHO; 2013.

10. Sarno F, Claro RM, Levy RB, Bandoni DH, Monteiro CA. Estimated sodium intake for the Brazilian population, 2008-2009. Rev Saúde Pública 2013; 47(3):571578.

11. Mill JG, Malta DC, Machado IE, Pate A, Pereira CA, Jaime PC, Szwarcwald CL, Rosenfeld LG. Estimativa do consumo de sal pela população brasileira: resultado da Pesquisa Nacional de Saúde 2013. Rev Bras Epidemiol 2019; 22(Supl. 2):E190009.SUPL.2.

12. Claro RM, Linders H, Ricardo CZ, Legetic B, Campbell NRC. Consumer attitudes, knowledge, and behavior related to salt consumption in sentinel countries of the Americas. Rev Panam Salud Publica 2012; 32(4):265-273.

13. Oliveira MM, Malta DC, Santos MAS, Oliveira TP, Nilson EAF, Claro RM. Consumo elevado de sal autorreferido em adultos: dados da Pesquisa Nacional de Saúde, 2013. Epidemiol Serv Saúde 2015; 24(2):249256. 
14. Brasil. Ministério da Saúde (MS). Plano de Ações Estratégicas para o Enfrentamento das Doenças Crônicas Não Transmissíveis (DCNT) no Brasil 2011-2022. Brasília: MS; 2011.

15. Szwarcwald CL, Malta DC, Souza JPRB, Almeida WS, Damacena GN, Pereira CA, Rosenfeld LG. Exames laboratoriais da Pesquisa Nacional de Saúde: metodologia de amostragem, coleta e análise dos dados. Rev Bras Epidemiol 2019; 22(Supl. 2):E190004.SUPL.2.

16. Malta DC, Szwarcwald CL, Silva JJB. Primeiros resultados da análise do laboratório da Pesquisa Nacional de Saúde. Rev Bras Epidemiol 2019; 22(Supl. 2):E190001.SUPL.2.

17. Tanaka T, Okamura T, Miura K, Kadowaki T, Ueshima $\mathrm{H}$, Nakagawa $\mathrm{H}$, Hashimoto T. A simple method to estimate populational 24-h urinary sodium and potassium excretion using a casual urine specimen. J Hum Hypertens 2002; 16(2):97-103.

18. Mill JG, Rodrigues SL, Baldo MP, Malta DC, Szwarcwald CL. Estudo de validação das equações de Tanaka e de Kawasaki para estimar a excreção diária de sódio através da coleta da urina casual. Rev Bras Epidemiol 2015; 18(Supl. 2):224-237.

19. World Health Organization (WHO). Obesity: preventing and managing the global epidemic. Geneva: WHO; 2000.

20. The International Expert Committee. International expert committee report on the role of the Alc assay in the diagnosis of diabetes. Diabetes Care 2009; 32(7):1327-1334.

21. World Health Organization (WHO). Use of glycated haemoglobin $(\mathrm{HbAlc})$ in the diagnosis of diabetes mellitus. Geneva: WHO; 2011.

22. Malta DC, Santos NB, Perillo RD, Szwarcwald CL. Prevalence of high blood pressure measured in the Brazilian population, National Health Survey, 2013. São Paulo Med J 2016; 134(2):163-170.

23. Expert Panel on Detection, Evaluation, and Treatment of High Blood Cholesterol in Adults. Executive summary of the third report of the National Cholesterol Education Program (NCEP) expert panel on detection, evaluation, and treatment of high blood cholesterol in adults (Adult Treatment Panel III). JAMA 2001; 285(19):2486-2497.

24. Kidney Disease Improving Global Outcomes. KDIGO 2012 Clinical Practice Guideline for the Evaluation and Management of Chronic Kidney Disease. Kidney Int Suppl 2013; 3(1):1-150.

25. World Health Organization (WHO). Haemoglobin concentrations for the diagnosis of anaemia and assessment of severity. Vitamin and Mineral Nutrition Information System. Genebra: WHO; 2011.

26. Brown IJ, Dyer AR, Chan Q, Cogswell ME, Ueshima $\mathrm{H}$, Stamler J, Elliott P. Estimating 24-h urinary sodium excretion from casual urinary sodium concentrations in western populations: The Intersalt Study. Am J Epidemiol 2013; 177(11):1180-1192.

27. Elliott P, Marmot M, Dyer A, Joossens J, Kesteloot H, Stamler R, Stamler J, Rose G. The INTERSALT study: main results, conclusions and some implications. Clin Exp Hypertens A 1989; 11(5-6):1025-1034.
28. Lim SS, Vos T, Flaxman AD, Danaei G, Shibuya K, Adair-Rohani H, Amann M, Anderson HR, Andrews KG, Aryee M, Atkinson C, Bacchus LJ, Bahalim AN, Balakrishnan K, Balmes J, Barker-Collo S, Baxter A, Bell ML, Blore JD, Blyth F, Bonner C, Borges G, Bourne R, Boussinesq M, Brauer M, Brooks P, Bruce NG, Brunekreef B, Bryan-Hancock C, Bucello C, Buchbinder R, Bull F, Burnett RT, Byers TE, Calabria B, Carapetis J, Carnahan E, Chafe Z, Charlson F, Chen H, Chen JS, Cheng AT-A, Child JC, Cohen A, Colson KE, Cowie BC, Darby S, Darling S, Davis A, Degenhardt L, Dentener F, Des Jarlais DC, Devries K, Dherani M, Ding EL, Dorsey ER, Driscoll T, Edmond K, Ali SE, Engell RE, Erwin PJ, Fahimi S, Falder G, Farzadfar F, Ferrari A, Finucane MM, Flaxman S, Fowkes FGR, Freedman G, Freeman MK, Gakidou E, Ghosh S, Giovannucci E, Gmel G, Graham K, Grainger R, Grant B, Gunnell D, Gutierrez HR, Hall W, Hoek HW, Hogan A, Hosgood 3rd HD, Hoy D, Hu H, Hubbell BJ, Hutchings SJ, Ibeanusi SE, Jacklyn GL, Jasrasaria R, Jonas JB, Kan H, Kanis JA, Kassebaum N, Kawakami N, Khang Y-H, Khatibzadeh S, Khoo J-P, Kok C, Laden F, Lalloo R, Lan Q, Lathlean T, Leasher JL, Leigh J, Li Y, Lin JK, Lipshultz SE, London S, Lozano R, Lu Y, Mak J, Malekzadeh R, Mallinger L, Marcenes W, March L, Marks R, Martin R, McGale P, McGrath J, Mehta S, Mensah GA, Merriman TR, Micha R, Michaud C, Mishra V, Hanafiah KM, Mokdad AA, Morawska L, Mozaffarian D, Murphy T, Naghavi M, Neal B, Nelson PK, Nolla JM, Norman R, Olives C, Omer SB, Orchard J, Osborne R, Ostro B, Page A, Pandey KD, Parry CDH, Passmore E, Patra J, Pearce N, Pelizzari PM, Petzold M, Phillips MR, Pope D, Pope 3rd CA, Powles J, Rao M, Razavi H, Rehfuess EA, Rehm JT, Ritz B, Rivara FP, Roberts T, Robinson C, Rodriguez-Portales JA, Romieu I, Room R, Rosenfeld LC, Roy A, Rushton L, Salomon JA, Sampson U, Sanchez-Riera L, Sanman E, Sapkota A, Seedat S, Shi P, Shield K, Shivakoti R, Singh GM, Sleet DA, Smith E, Smith KR, Stapelberg NJC, Steenland K, Stöckl H, Stovner LJ, K Straif, Straney L, Thurston GD, Tran JH, Van Dingenen R, van Donkelaar A, Veerman JL, Vijayakumar L, Weintraub R, Weissman MM, White RA, Whiteford H, Wiersma ST, Wilkinson JD, Williams HC, Williams W, Wilson N, Woolf AD, Yip P, Zielinski JM, Lopez AD, Murray CJL, Ezzati M, AlMazroa MA, Memish ZA. A comparative risk assessment of burden disease and injury attributable to 67 risk factor clusters in 21 regions, 1990-2010: a systematic review for the Global Burden of Disease Study 2010. Lancet 2012; 380(9859):2224-2260.

29. He FJ, Li J, MacGregor GA. Effect of longer term modest salt reduction on blood pressure: Cochrane systematic review and meta-analysis of randomized trials. BMJ 2013; 346:f1325.

30. McLean RM. Measuring population sodium intake: A review of methods. Nutrients 2014; 6(11):4651-4662.

31. Campbell NRC, He FJ, Tan M, Cappuccio FP, Neal B, Woodward M, Cogswell ME, McLean R, Arcand J, MacGregor G. The international consortium for quality research on dietary sodium/salt (TRUE) position statement on the use of 24-hour, spot, and short duration $(<24$ hours) timed urine collections to assess dietary sodium intake. J Clin Hypertens (Greenwich) 2019; 21(6):700-709. 
32. Xu J, Zhang J, Liu M, Bai Y, Guo X, Dong J, Xu A, Wu J. Estimating 24-hour sodium excretion from spot urine samples in Chinese adults: Can spot urine substitute 24-hour urine samples? Nutrients 2020; 12(3):798.

33. Mill JG, Silva AB, Baldo MP, Molina MC, Rodrigues SL. Correlation between sodium and potassium excretion in 24- and 12-h urine samples. Braz J Med Biol Res 2012; 45(9):799-805.

34. Henry JP. Stress, salt and hypertension. Soc Sci Med 1988; 26(3):293-302.

35. Oparil S. Low sodium intake - Cardiovascular health benefit or risk? N Engl J Med 2014; 371:677-679.

36. GBD 2017 Risk Factor Collaborators. Global, regional, and national comparative risk assessment of 84 behavioural, environmental and occupational, and metabolic risks or clusters of risks for 195 countries and territories, 1990-2017: a systematic analysis for the Global Burden of Disease Study 2017. Lancet 2018; 392(10159):1923-1994.

37. Mente A, O’Donnell MJ, Rangarajan S, McQueen MJ, Poirier P, Wielgosz A, Morrison H, Li W, Wang X, Di C, Mony P, Devanath A, Rosengren A, Oguz A, Zatonska K, Yusufali AH, Lopez-Jaramillo P, Avezum A, Ismail N, Lanas F, Puoane T, Diaz R, Kelishadi R, Iqbal R, Yusuf R, Chifamba J, Khatib R, Teo K, Yusuf $S$, PURE Investigators. Association of urinary sodium and potassium excretion with blood pressure. New Engl J Med 2014; 371(7):601-611.

38. Malta DC, Bernal RTI, Andrade SSCA, Silva MMA, Velasquez-Melendez G. Prevalência e fatores associados com hipertensão arterial autorreferida em adultos brasileiros. Rev Saúde Pública 2017; 51(Supl. 1):11s.

39. Nerbass FB, Pecoits-Filho R, McIntyre NJ, McIntyre CW, Taal MW. High sodium intake is associated with important risk factors in a large cohort of chronic kidney disease patients. Eur J Clin Nutr 2015; 69(7):786-790.

40. Rodrigues SL Souza Júnior, PR, Pimentel EB, Baldo MP, Malta DC, Mill JG, Szwarcwald CL. Relationship between salt consumption measured by 24 -h urine collection and blood pressure in the adult population of Vitória (Brazil). Braz J Med Biol Res 2015; 48(8):728-735.

41. Instituto Brasileiro de Geografia e Estatística (IBGE). Aquisição alimentar domiciliar per capita. Rio de Janeiro: IBGE; 2010.

42. Nilson EAF, Spaniol AM, Gonçalves VSS, Moura I, Silva SA, L'Abbé M, Jaime PC. Sodium reduction in processed foods in Brazil: Analysis of food categories and voluntary targets from 2011 to 2017. Nutrients 2017; 9(7):742.

43. Simões BDS, Barreto SM, Molina MDCB, Luft VC, Duncan BB, Schmidt MI, Benseñor IJM, Cardoso LO, Levy RB, Giatti L. Consumption of ultra-processed foods and socioeconomic position: a cross-sectional analysis of the Brazilian Longitudinal Study of Adult Health (ELSA-Brasil). Cad Saúde Pública 2018; 34(3):e00019717.

44. Instituto Brasileiro de Geografia e Estatística (IBGE). Pesquisa de orçamentos familiares 2017-2018: avaliação nutricional da disponibilidade domiciliar de alimentos no Brasil. Rio de Janeiro: IBGE; 2020.
45. Grimes CA, Wright JD, Liu K, Nowson CA, Loria CM. Dietary sodium intake is associated with total fluid and sugar-sweetened beverage consumption in US children and adolescents aged 2-18 y: NHANES 20052008. The Am J Clin Nutrition 2013; 98(1):189-196.

46. Ma Y, He FJ, MacGregor GA. High salt intake: Independent risk factor for obesity? Hypertension 2015 66(4):843-849.

47. Nakandakare ER, Charf AM, Santos FC, Nunes VS, Ortega K, Lottenberg AMP, Mion Jr D, Nakano T, Nakajima K, D’Amico EA, Catanozi S, Passarelli M, Quintão ECR. Dietary salt restriction increases plasma lipoprotein and inflammatory marker concentrations in hypertensive patients. Atherosclerosis 2008; 200(2):410-416.

48. Sarno F, Jaime PC, Ferreira SRG, Monteiro CA. Consumo de sódio e síndrome metabólica: uma revisão sistemática. Arq Bras Endocrinol Metab 2009; 53(5):608-616.

49. Zanini RV, Araújo CL, Martínez-Mesa J. Utilização de adoçantes dietéticos entre adultos em Pelotas, Rio Grande do Sul, Brasil: um estudo de base populacional. Cad. Saúde Pública 2011; 27(5):924-934.

50. Oliveira ALM. Comparação do teor de sódio em produtos industrializados diet e light e seus análogos convencionais. Nutrição Brasil 2017; 16(1):29-36.

51. Kim MK. Dietary sodium intake in patients with type 2 diabetes mellitus. Diabetes Metab J 2016; 40(4):280282.

52. Martyn D, Darch M, Roberts A, Lee H Y, Tian TY, Kaburagi N, Belmar P. Low-/no-calorie sweeteners: A review of global intakes. Nutrients 2018; 10(3):357.

53. Pase MP, Himali JJ, Beiser AS, Aparicio HJ, Satizabal CL, Vasan RS, Seshadri S, Jacques PF. Sugar- and artificially sweetened beverages and the risks of incident stroke and dementia: A prospective cohort study. Stroke 2017; 48(5):1139-1146.

54. Malta DC, Morais Neto OL, Silva Junior JB. Apresentação do plano de ações estratégicas para o enfrentamento das doenças crônicas não transmissíveis no Brasil, 2011 a 2022. Epidemiol Serv Saúde 2011; 20(4):425-438.

55. World Health Organization (WHO). Global Action Plan for the Prevention and Control of NCDs 20132020. Genebra: WHO; 2013.

56. Brasil. Ministério da Saúde (MS). Secretaria de Atenção à Saúde. Secretaria de Atenção à Saúde. Departamento de Atenção Básica. Guia alimentar para a população brasileira. 2a ed. Brasília: MS; 2014.

Artigo apresentado em 04/10/2020

Aprovado em 23/10/2020

Versão final apresentada em 25/10/2020

Editores chefes: Maria Cecília de Souza Minayo, Romeu Gomes, Antônio Augusto Moura da Silva 
